\title{
An Object Representation System Using Virtual Space Coordinates
}

\author{
Kyeong-Jin Ban, Jong-Chan Kim and Eung-Kon Kim, Member, KIMICS
}

\begin{abstract}
Conventional Augmented Reality has used data gloves or markers for smooth interaction between objects and background. This causes inconvenience of use and lower immersion. To build up immersion in Augmented Reality, additional input devices must be removed. For this purpose, accurate recognition of space coordinates is needed even with no attachment of markers. This paper proposes a method to create virtual space coordinates for interaction without wearing additional input devices so as to improve immersion in Augmented Reality. The acquired image was projected to $2 \mathrm{D}$ space and vanishing lines were extracted to calculate the virtual space coordinates. Then the sizes of the inserted objects were varied in accordance with the size of the virtual coordinates area based on the image projected onto the 2D coordinates. This resulted in improved immersion. This method can increase the efficiency of object creation by excluding the use of a 3D modeler for creation of 3D objects.
\end{abstract}

Index Terms- Augmented reality, Vanishing lines, Difference image, Projection, Mapping

\section{INTRODUCTION}

With the development of computer graphics technology, virtual reality (VR) is being widely used in various contents such as movies and games. Augmented reality which is a type of VR is gradually spreading into the domain of actual life. Augmented reality is a technology located between VR and reality such as images, and provides a seamless mixture of real world and virtual world in real time for the experience of enhanced immersion and sense of reality by users [1]. Augmented reality improves the sense of reality by adding virtual information to the actual environment of user. Furthermore, the 3D representation of Augmented Reality improves understanding of the phenomena in a physical space. In particular, Augmented Reality widens and deepens the understanding of phenomena by providing various points of view including the first person point of view that reinforces direct experience and the third person point of view that assists understanding of phenomena from the omniscient perspective. In addition to simulation of the real world,

\footnotetext{
Manuscript received July 1, 2010; accepted July 1, 2010.

Kyeong-Jin Ban is with the Department of Computer Science, Sunchon National University, 413 jungangno Suncheon, 540-742, Korea (Email: multwave@sunchon.ac.kr) Jong-Chan Kim(Email: seaghost@sunchon.ac.kr) Corresponding Author : Eung-Kon Kim(Email: kek@sunchon.ac.kr)
}

Augmented Reality provides the benefit of actualizing experiences that are impossible in real world using various sense organs with virtual objects. However, there are limitations to the use of Augmented Reality in real environment due to the space arrangement technology that uses markers to facilitate virtual information in real environment as well as the limited technology for 3D object creation. To solve such problems of space arrangement technology and object creation technology, many studies are being conducted on markerless augmentation and object creation methods. Markers have been used in Augmented Reality to represent and recognize $3 \mathrm{D}$ coordinates in an environment. However, creating 3D objects on 3D coordinates and arranging objects to recognized locations increases the burden on the system. Therefore, this paper proposes a method to represent $3 \mathrm{D}$ objects on virtual 2D space coordinates mapped to the real environment for creation and augmentation of 3D objects.

Chapter II of this paper explains VR and Augmented Reality in more detail; Chapter III describes the method to represent $3 \mathrm{D}$ objects in a $2 \mathrm{D}$ space; Chapter IV describes the result of inserting 3D objects onto virtual 2D space coordinates; and finally Chapter V summarizes conclusions.

\section{RELATED WORK}

\section{A. Augmented Reality}

Augmented Reality is a technology for showing virtual objects overlapped with the real world as viewed from user's eyes. Because the virtual world that contains additional information is mixed with the real world in real time, it is also called Mixed Reality (MR).

Augmented Reality was first studied by Ivan Sutherland as a development of the see-through HMD (head mounted display). The definition of Augmented Reality by Ronald Azuma best explains its characteristics. He mentions a few elements of the Augmented Reality's goal, and we can understand the overall meaning of Augmented Reality. According to the definition by Azuma, Augmented Reality is a mixture of real-world elements and the images of virtual reality which allows real-time interaction.

Augmented Reality is an area of VR but has a meaning different from VR. VR technology makes users immerse 
in a virtual environment and they cannot see the real environment. However, Augmented Reality that mixes real environment with virtual objects allows users to see the real environment to provide higher sense of reality and additional information. Research and development on Augmented Reality as a hybrid VR system that combines real environment with virtual environment has been active since late 1990s around the USA and Japan.

Recently, some mobile devices show 3D images with the locations of shops and telephones when user captures his/her surroundings with the built-in camera. With the recent propagation of smart phones, Augmented Reality has entered the stage of full-scale commercialization. Many products using Augmented Reality are now being developed including games, mobile solutions, and educational solutions.

The implementation technologies for Augmented Reality can be applied to a wide variety of real-world environment such as location positioning, biometrics, artificial intelligence, implementation of five senses, mobile technology, and in particular, the next-generation display technology that is ideal for the ubiquitous environment.

\section{B. Depth Creation Using Vanishing Point}

Vanishing points are powerful clues for depth perception in indoor or outdoor environment with artificial structures. A vanishing point corresponds to the point at the farthest distance from observer. Human beings can estimate vanishing point using the geometrical components of a single-eye image, and perceive relative depth based on the location of the vanishing point and the viewpoint of the observer.

Vanishing point is one of the depth clues used to solve the problem of restoring the $3 \mathrm{D}$ spatial structure from $2 \mathrm{D}$ images. Images that contain artificial structures such as buildings, roads, and railroads consist of geometrical elements such as straight lines, and include many parallel or orthogonal straight lines. The distance between two parallel lines in a 3D space becomes shorter as they get farther from the observer in a projected image on a 2D plane and ultimately meet at one point. This point is called a vanishing point and the lines that meet at the vanishing point are called vanishing lines. Vanishing points are very powerful clue for depth in outdoor images that contain architectural structures such as buildings and roads and indoor images such as corridor and room [2],[3]. As vanishing points are generally on the horizon, they represent the farthest point from the bottom of the image. Thus, it is possible to estimate the relative depth using vanishing points.

As a method for detecting vanishing point using straight lines, the Gaussian sphere is used [4]. Figure 1 illustrates the detection of vanishing point using the Gaussian sphere.

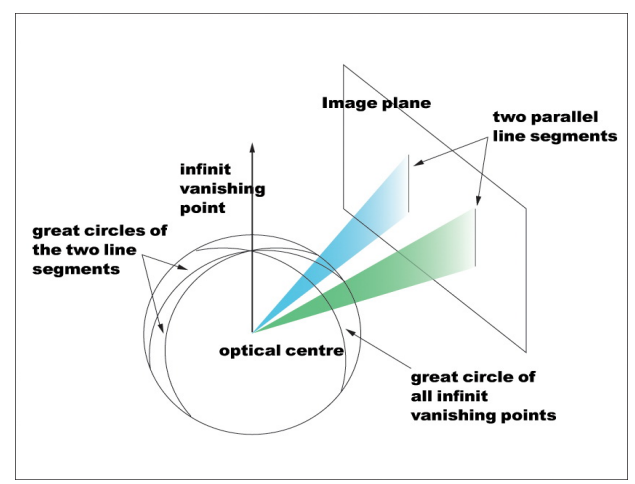

Fig. 1 Gaussian sphere for detecting vanishing point

\section{OBJECT REPRESENTATION METHOD USING VIRTUAL SPACE COORDINATES}

\section{A. Creation of $2 D$ Coordinates and Extraction of Vanishing Lines}

In order to use Augmented Reality, we must capture the real environment and generate space coordinates on the basis of the captured images, and then add information and images to the generated coordinates. In general, when the environment is captured horizontally to the bottom, the bottom does not go over the center of the picture. The bottom and top are divided by the center through the environment capturing.

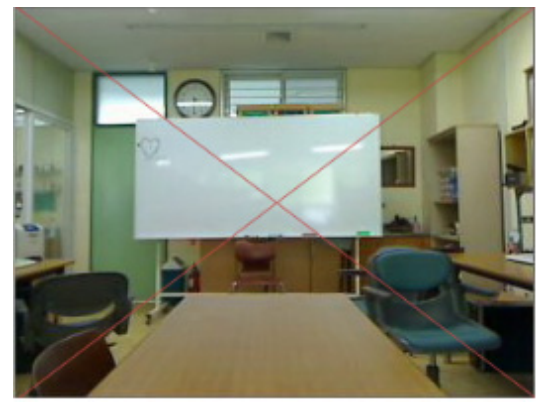

(a) Photographing of the background and establishment of the reference lines

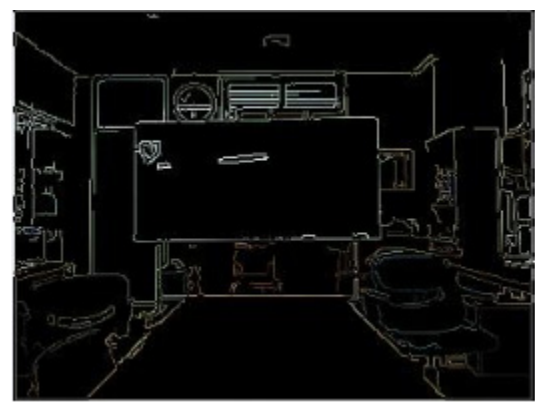

(b) Vanishing lines extracted by edge extraction

Fig. 2 Generation of vanishing lines through background capturing and edge extraction 
Radial coordinates are set at fixed angles around the central axis of the image in the virtual space based on the image size. These coordinates are synthesized with the characteristics of the captured image to create a screen with radial coordinates generated at fixed angles based on the image size. Figure 2 illustrates the generation of vanishing lines through background capturing and edge extraction. For extraction of vanishing lines, the capturing environment was limited to an office and parallel lines toward the center were extracted trough edge extraction from the original image. The vanishing lines extracted by image processing were mapped to the virtual coordinates that have been generated to create coordinates in the virtual space.

\section{B. Object Extracting Method on Virtual Coordinates}

To represent objects on 2D coordinates, when an entity for object augmentation is added to the background, it is extracted through a difference image compared to the original background. The difference image means the difference in brightness of pixels corresponding to the same locations in two images [5]. A binary image is created from two gray scale images which has been binarized for random threshold values.

The difference image can be obtained by the following equations:

$$
\begin{aligned}
& \delta I(x, y)=\left|I_{t}(x, y)-I_{t-1}(x, y)\right| \\
& D(x, y)=\left\{\begin{array}{lc}
1, & \text { if } \delta I(x, y)>T_{h} \\
0, & \text { otherwise }
\end{array}\right.
\end{aligned}
$$

In Equation (1), $\delta I(x, y)$ is the difference in brightness of the pixels at the coordinate $(x, y), I_{t}(x, y)$ is the current image, and $I_{t-1}(x, y)$ is the previous image.

The object size on the space coordinates is determined through mapping of the extracted entity to the space coordinates, and the object size is varied by distance difference for augmentation to the entity. Figure 3 represents the image extracted through difference image by object distance and the size of the area occupied by the object.

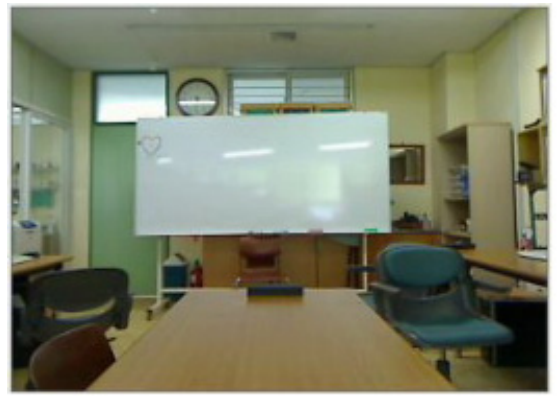

(a) Original image of object at long distance

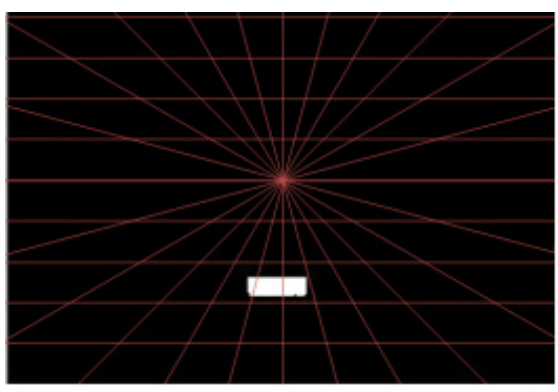

(b) Result of object extraction at long distance

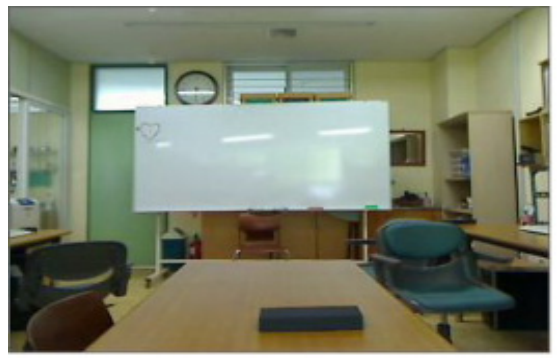

(c) Original image of object at short distance

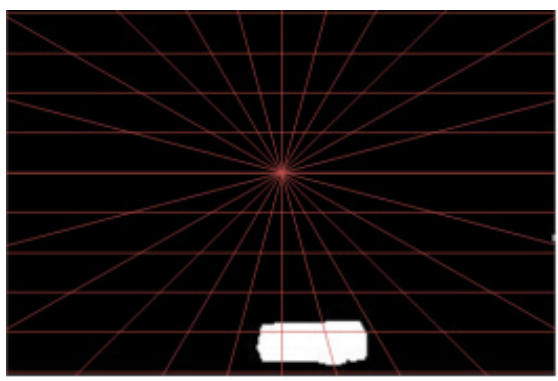

(d) Result of object extraction at short distance

Fig. 3 Extraction Results by Object Distance

\section{AUGMENTED REALITY SYSTEM USING VIRTUAL SPACE COORDINATES}

\section{A. Markerless Augmented Reality System and Implementation Environment}

Augmented Reality shows real-world images overlapped with virtual graphics. To obtain accurate images, virtual objects must be drawn at the desired locations on the screen. Traditionally, 3D coordinates were generated through markers for efficient augmentation. However, the marker system is cumbersome to use and poses environmental limitations. In general, images output to a monitor screen, a $3 \mathrm{D}$ space is projected onto a $2 \mathrm{D}$ space. This means that satisfactory augmentation effects can be obtained from virtual coordinates of images projected on the $2 \mathrm{D}$ space. $2 \mathrm{D}$ coordinates were generated on the basis of the 2D images projected on the monitor screen, which were projected on the extracted entities. Figure 4 shows a flowchart for Augmented Reality using the proposed virtual coordinates.

This system is for Markerless augmentation which was 
implemented using CPU Intel Core2 Quad Q9400, VGA NVIDIA Quadro FX 3700, RAM 6GB, WabCam 604X480 Pixels, Visual C++ 6.0 and OpenCV and JPEG images were used as the virtual object.

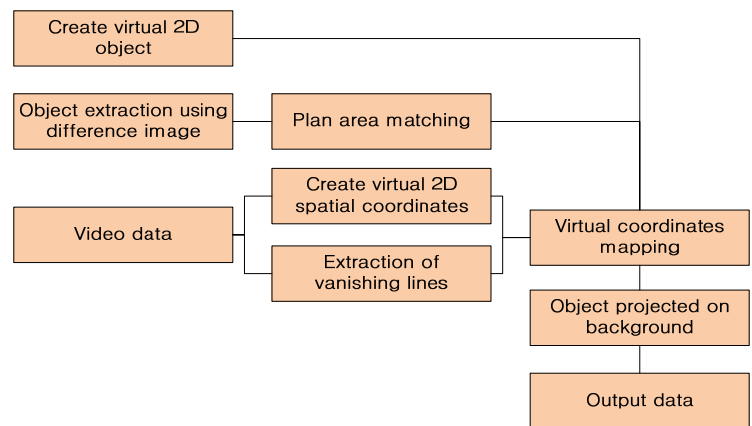

Fig. 4 Flowchart of Augmented Reality System Using Virtual Space Coordinates

\section{B. 2D Virtual Space Coordinates Mapping and Projection}

This study used images of the resolution of $640 \times 480$ pixels. The captured image was divided into 24 zones from the center in intervals of 15 degrees, and 10 horizontal zones were added to express depth. In this way, 96 planes were generated by the lines derived from the horizontal line and the center line.

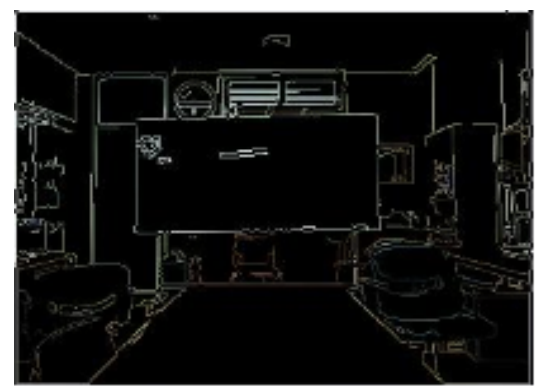

(a) Vanishing lines extracted by edge extraction

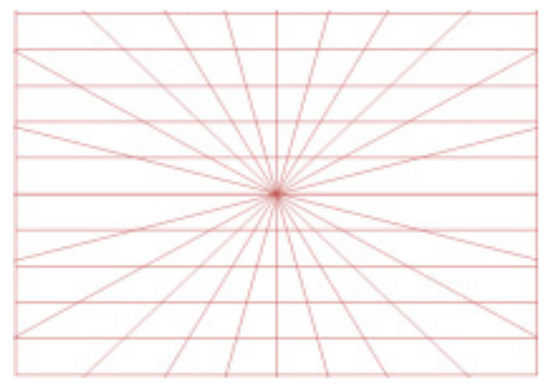

(b) Virtual coordination

Fig. 5 Extraction of Vanishing Lines and Setting of Virtual Coordinate Zones

Among the 96 virtual zones, 48 zones that correspond to the bottom were used for projection of virtual objects. The 96 zones used the angles of the vanishing lines extracted from the original image. Figure 5(a) shows the vanishing lines extracted by edge extraction from the original image. The vanishing lines are radiated in intervals of approximately 135 degrees from the center of the image and the reference lines which are radiated in intervals of 15 degrees are created in a 2D space as shown in Figure 5(b).

Shade effects were applied to the 2D virtual objects to give the sense of three dimensions. The virtual objects were augmented in proportion to the plane area size of the entities and the virtual coordinates extracted through difference image in a $2 \mathrm{D}$ space of virtual coordinates. Because the plane size of the augmented objects varies by the distance on the virtual coordinates, the size of the augmented objects is in inverse proportion to the distance.

\section{CONCLUSIONS}

This paper proposed a method for creating virtual space coordinates to enable interaction without additional input devices for improved immersion in Augmented Reality. The acquired image was projected to a $2 \mathrm{D}$ space, and vanishing lines were extracted for generation of virtual space coordinates. When an entity to be augmented is inserted to the background, it was extracted through a difference image compared to the original background, and the object was augmented in proportion to the plane area size of the entity and virtual coordinates.

For virtual object augmentation, a method to arrange objects based on the image projected on a 2D screen was proposed to solve the problem of arranging objects on 3D coordinates. This method can increase the efficiency of object creation by excluding the use of a 3D modeler to create 3D objects. Furthermore, immersion was improved by varying the size of objects inserted by the plane size of virtual coordinates based on the image projected on 2D coordinates. The results of this study can be applied to markerless augmentation and mobile augmentation.

Figure 6 shows the object augmentation result by captured image and distance.

In the future, we need to study on a method for creating virtual coordinates according to the moving camera viewpoint. Furthermore, a study on a method to improve immersion and sense of three dimensions is needed.

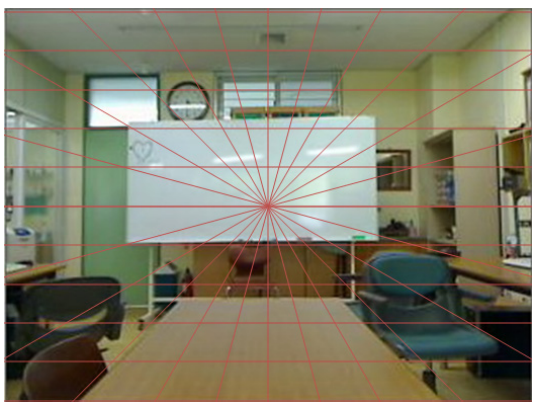

(a) Background and virtual coordinates mapping 


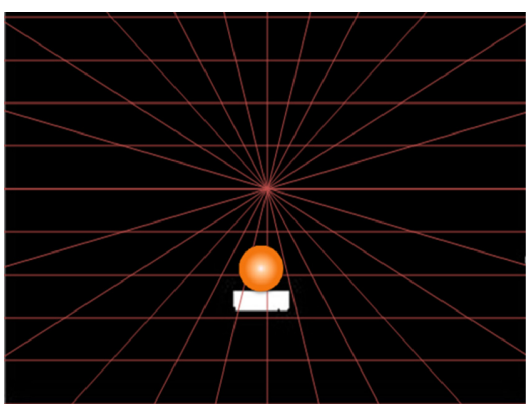

(b) Result of object extraction and mapping at long distance

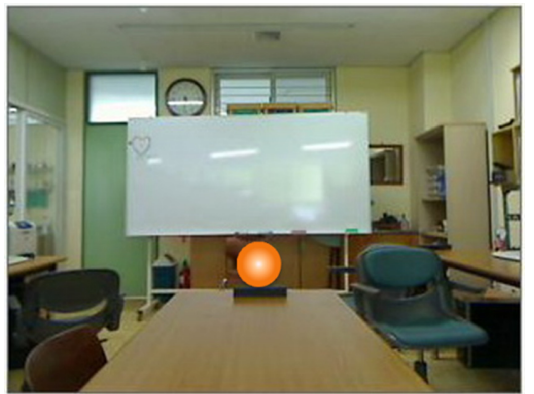

(c) Object augmentation result at long distance

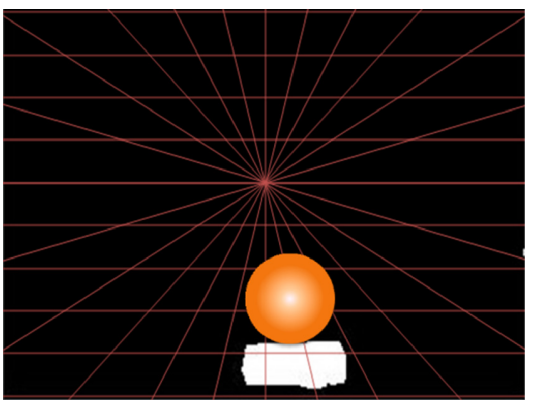

(d) Result of object extraction and mapping at short distance

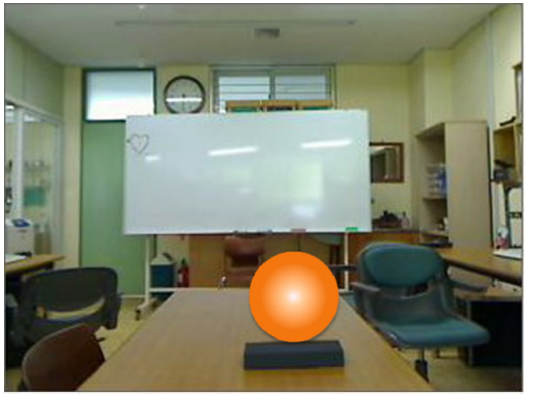

(e) Object augmentation result at short distance

Fig. 6 Object Augmentation Results by Captured Image and Distance

\section{ACKNOWLEDGMENT}

This research was supported by the MKE(The Ministry of Knowledge Economy), Korea, under the ITRC(Information Technology Research Center) support program supervised by the NIPA(National IT Industry Promotion Agency) (NIPA-2010-C1090-1021-0012)

\section{REFERENCES}

[1] R. T. Azuma, "A survey of Augmented Reality," In presence: Teleoperators and Virtual Environment, vol.6, no.4, pp.355-385, Aug 1997.

[2] C. Rother, "A New Approach for Vanishing Point Detection in Architectural Environments," IVC 2002, 20(9-10):647-656, 2002.

[3] Jana KoseckáWei Zhang, "Efficient Computation of Vanishing Points," ICRA 2002: 223-228, 2002.

[4] J.A. Shufelt, "Performance Evaluation and Analysis of Vanishing Point Detection Techniques," Proc. ARPA Image Understanding Workshop, pp.1,113-1,132, Feb 1996.

[5] M.K. Oh, K.H. Han, D.J and B.M.Jun, "Moving object tracking using differential image," Korea Contents Association Conference, vol. 2, no.1, pp. 396-400, May 2004.

[6] A.D. Cheok, K.H. Goh, W Liu, F. Farbiz, S.L. Teo, H.S.Teo, S. P. Lee, Y.Li, S.W.Fong and X.Yang, "Human Pacman: a mobile widearea entertainment system based on physical, social, and ubiquitous computing," Springer Trans. Personal and Ubiquitous Computing, vol.8, no.2, pp.71-81, May 2004.

[7] R. Grasset, A. Duenser and M. Billinghurst, "Human-Centered Development of an AR Handheld Display," Proc. of ISMAR, pp.14, 2007.

[8] P. Grimm, M. Haller, V. Paelke, and S. Reinhold, "AMIRE Authoring Mixed Reality," The First IEEE International Augmented Reality Toolkit Workshop, Sept 2002.

[9] D. Hoiem, A.A. Efros, and M. Hebert, "Automatic Photo Pop-up," ACM SIGGRAPH, 2005.
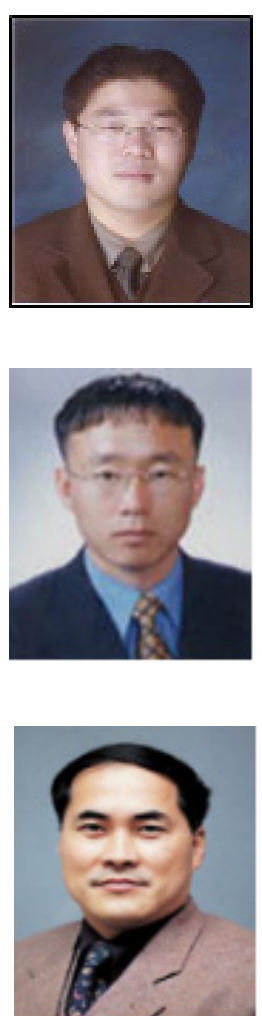

computer graphics, multimedia and HCI.

\section{Kyeong-Jin Ban}

$\mathrm{He}$ received his B.S. degree from Sunchon National University in 2003, his M.S degree from department of computer science, Sunchon National University in 2005, his Ph.D degree from department of computer science, Sunchon National University in 2007. His current research interests are computer graphics, image processing, HCI, VR and AR.

\section{Jong-Chan Kim}

$\mathrm{He}$ received his B.S. degree from Sunchon National University in 2000 , his M.S degree from depart-ment of computer science, Sunchon National University in 2002, his Ph.D. degree from department of computer science, Sunchon National University in 2007. His current research interests are image processing, computer graphics, AR, games and $\mathrm{HCI}$.

\section{Eung-Kon Kim}

He received B.E. degree Cho-sun University in 1980, his M.E. degree from depar-tment of computer engineering, Hanyang University in 1986, his Ph.D degree form depart-ment of computer engineering, Chosun University in 1994, In 2010, he joined department of computer science, the Sunchon National University, where he is a professor. His current research interests are image processing, AR, 